\title{
Are there specific metacognitive processes associated with anxiety disorders in youth?
}

This article was published in the following Dove Press journal:

Psychology Research and Behavior Management

16 September 2010

Number of times this article has been viewed

\section{Terri Landon Bacow' \\ Jill Ehrenreich May ${ }^{2}$ \\ Leslie R Brody ${ }^{3}$ \\ Donna B Pincus ${ }^{4}$}

'Mount Sinai School of Medicine, New York City, NY, USA; '2Department of Psychology, University of Miami, FL, USA; ${ }^{3}$ Department of Psychology, ${ }^{4}$ Center for Anxiety and Related Disorders, Boston University, MA, USA
Correspondence: Terri L Bacow Mount Sinai School of Medicine, One Gustave L Levy Place, Box I230,

New York, NY 10029, USA

Tel +l 9177100846

Email terri.bacow@msssm.edu

\begin{abstract}
While Wells' metacognitive model of generalized anxiety disorder (GAD) posits that certain metacognitive processes, such as negative meta-worry (negative beliefs about worry), are more strongly associated with symptoms of GAD than other anxiety disorders in adults, research has yet to determine whether the same pattern is true for younger individuals. We examined the relationship between several metacognitive processes and anxiety disorder diagnostic status in a sample of 98 youth aged 7-17 years. Twenty youth with GAD were compared with similarly sized groups of youth with obsessive-compulsive disorder (OCD, $\mathrm{n}=18)$, social phobia (SOC, $n=20)$, separation anxiety disorder ( $\mathrm{SAD}, \mathrm{n}=20$ ), and healthy controls who were not patients (NONP, $n=20$ ) using a self-report measure of metacognition adapted for use with young people in this age range (Metacognitions Questionnaire for Children). Contrary to expectations, only one specific metacognitive process was significantly associated with an anxiety disorder diagnosis, in that the controls endorsed a greater degree of cognitive monitoring (self-reported awareness of one's thoughts) than those with SAD. In addition, there was a trend indicating that nonpatients scored higher than youth with GAD on this scale. These surprising results suggest potentially differing patterns in the relationships between symptoms and metacognitive awareness in anxious youth, depending on the type of anxiety disorder presentation.
\end{abstract}

Keywords: metacognition, childhood, adolescence, anxiety, diagnosis

\section{Introduction}

Wells and colleagues' model of adult internalizing disorders differs from other etiological frameworks in that it highlights the importance of metacognition in the development of anxiety and mood symptoms. ${ }^{1}$ More specifically, Wells' model of metacognition explores the role of individuals' beliefs and perceptions about their own cognitive processes in the emergence of pathological worry states, such as generalized anxiety disorder (GAD). According to Wells, metacognitive knowledge refers to beliefs that individuals have about their own cognitions, including the ideas people have about the meanings of particular types of thoughts. ${ }^{2}$ The author further argues that such beliefs are linked to emotional well-being. For example, while people generally believe that worrying can be advantageous, adults with GAD tend to believe that worrying is uncontrollable and dangerous, 3,4 and thus tend to score higher on measures of negative meta-worry (eg, negative beliefs about worry) than individuals without anxiety disorders. For individuals with of obsessive-compulsive disorder (OCD), beliefs about superstition, punishment and responsibility ("SPR" beliefs) and heightened cognitive self-consciousness (eg, heightened awareness of thoughts) seem to be more prevalent due to the salience assigned to these cognitions..$^{5-8}$ Further, Wells' metacognitive 
model of post-traumatic stress disorder (PTSD) suggests that both positive and negative metacognitive beliefs, along with thought control strategies, are associated with traumatic stress. ${ }^{9}$ These studies indicate that beliefs about the meaning, danger and power of thoughts may not be equivalent for all anxiety disorders.

To date, one study has examined the metacognitive beliefs of adults with different anxiety disorder diagnoses (GAD, panic disorder, social phobia and nonpatients), ${ }^{10}$ providing particular support for the metacognitive model of GAD. Results from this study suggested that there are diagnostic differences in levels of negative meta-worry by diagnostic category, but not in levels of positive meta-worry or SPR beliefs amongst individuals in this sample. The authors argued that negative meta-worry was a distinguishing factor in the comparison between GAD and other disorders, because GAD can be characterized as a high negative metacognition problem, while the other disorders are best discriminated from each other by their actual worry content. ${ }^{10}$ Wells \& Carter ${ }^{10}$ further found that distinguishing between the actual content of individuals' worries (eg, health, social concerns, referred to as "Type 1 worry") and meta-worry (eg, worry about worry, or "Type 2 worry") was helpful, because it allowed them to examine between-group differences in metaworry independently of the influence of worry about different content areas. In this study, the authors found that their ability to compare different diagnostic groups was improved when worry content was controlled, suggesting that meta-worry (as opposed to worry about different topics) truly accounted for diagnostic group differences.

Despite research documenting that cognitive processes are central components of anxiety in childhood and adolescence, ${ }^{11}$ only one study to date has explored whether similar patterns of metacognitive beliefs are present in younger individuals with and without differing anxiety disorders. ${ }^{12}$ Bacow et al $^{12}$ explored the metacognitive beliefs of a sample of clinically anxious $(n=78)$ and nonanxious youth $(n=20)$ aged $7-17$ years. The four metacognitive processes examined using a measure referred to as the Metacognitions Questionnaire for Youth (MCQ-C) ${ }^{12}$, a measure expanded from an adolescent version (Metacognitions Questionnaire for Adolescents, MCQ-A) $)^{13}$ to be applicable to a broader age range of youth, were positive meta-worry, negative meta-worry, SPR beliefs and cognitive monitoring (awareness of thoughts). Results of this study indicated that with youth's worry content controlled, negative meta-worry was significantly associated with child and adolescent self-reports of internalizing symptoms (excessive worry and depression). Age-based differences on the MCQ-C were found for cognitive monitoring only, with adolescents reporting greater awareness of their thoughts than children. Adolescent girls scored higher on the total scale of the measure than adolescent boys.

While this study suggested that metacognitive processes are endorsed by youth with and without anxiety disorders, the clinically anxious youth in this investigation did not score higher on the MCQ-C than the nonclinical controls. In fact, nonclinical youth endorsed a greater awareness of their thoughts than participants with anxiety disorders. The authors posited that this was perhaps due to the possibility that anxious youth wished to avoid focusing on or thinking about their anxious cognitions. ${ }^{13}$ They also suggested that youth without anxiety disorders may be able to freely focus their attention on nonworrisome thoughts because their attentional resources are not diverted to or consumed by threatening stimuli or anxious cognitions. ${ }^{14}$ Bacow et al ${ }^{12}$ noted that despite the lack of between-group differences, it would be beneficial to investigate whether any within-group differences found in this study, it would be beneficial to investigate whether any within-group differences in metacognitive processes exist between those with differing primary anxiety disorders in the clinical sample. This line of further inquiry would be helpful due to the absence of existing research exploring whether particular metacognitive processes are more frequently endorsed by youth with specific forms of anxiety disorders, and whether a metacognitive model of GAD (and other anxiety disorders) may be applicable to youth.

It appears plausible that, when compared with adults, youth with GAD may be more likely to have negative metaworries than youth with other anxiety disorder diagnoses, similar to the pattern observed in adulthood. In fact, in a study piloting the exploration of metacognitive beliefs in adolescents aged 13-17 years, Cartwright-Hatton et al ${ }^{13}$ found that a small clinical sample of adolescents (diagnosed with emotional disorders, with no specific diagnosis assigned) reported having a greater number of meta-worries about the danger of worrying than nonclinical participants, and Szabo and Lovibond ${ }^{15}$ also observed that children's worry episodes predominantly contained thoughts reflecting anticipation of negative outcomes. Furthermore, a study in China documented a positive relationship between meta-worry and GAD in a large group of middle-school students. ${ }^{16}$

Youth with OCD may also be more likely to exhibit hypervigilance with regards to their thoughts and interpret their thoughts in a superstitious manner (eg, SPR beliefs) when compared to youth with other anxiety diagnostic 
categories. Matthews et $\mathrm{al}^{17}$ explored the relationship between metacognitive beliefs and obsessional symptoms in a group of nonclinical adolescents aged 13-16 years. They found that metacognitive beliefs (measured by the MCQ-A) in general were strongly associated with higher levels of obsessive-compulsive symptoms and that when compared to thought-action fusion (eg, the belief that a thought is like an action), metacognitive beliefs and inflated responsibility (eg, perceived accountability for the outcome of intrusive thoughts) both emerged as significant independent predictors of OCD symptoms. Although they did not report findings from specific metacognitive subscales, further exploration of the data determined that both the SPR beliefs subscale and the cognitive self-consciousness (CSC) (a construct similar to awareness of thoughts) subscale of the MCQ-A had a significant positive correlation with the total score of the Leyton Obsessive-Compulsive Inventory-Child Version (S. Reynolds, personal communication with author, September 21, 2007). In a similar study with 126 nonclinical adolescents, ${ }^{18} \mathrm{CSC}$ was found to predict OCD symptoms after controlling for negative affect. Taken together, these findings suggest that the construct, cognitive awareness of thoughts, may have particular salience for youth with OCD.

The aim of the present study was to investigate potential differences in metacognitive processes amongst youth exhibiting differing anxiety disorders. Although GAD and OCD are two anxiety disorders that are particularly characterized by intrusive thought (and thus may result in greater selective attention to thought processes), from a clinical perspective, worry may also be viewed as a central component of other childhood anxiety disorders. For example, youth with social phobia (SOC) have frequent and intense worries about receiving negative evaluation from peers and other adults in social situations, and youth with separation anxiety disorder (SAD) may have severe worries that something bad will happen to their parents or themselves..$^{19}$ It is not clear whether a metacognitive theory of anxiety in youth is specific to GAD and $\mathrm{OCD}$, or is applicable to childhood anxiety disorders in which worry content (versus process) is more a central feature (eg, SAD and SOC).

In summary, our goal in this study was to explore whether any differences in metacognitive processes would be reported by a sample of youth with different anxiety disorder diagnoses (eg, GAD, OCD, SOC and SAD) versus a control group of nonpatients, and whether these differences would be upheld when the excessive nature and content of worry thoughts was controlled. In this paper, we report additional analyses conducted with the sample from our initial investigation ${ }^{12}$ exploring these questions. Thus, the same four metacognitive processes were selected for examination based on previous research: positive and negative meta-worry, SPR beliefs, and cognitive monitoring. We hypothesized that for youth in this sample, increased negative meta-worry would be more frequently endorsed by youth with GAD and that increased cognitive monitoring and SPR beliefs would be more commonly indicated by youth with OCD, as compared to other anxiety diagnoses and nonpatients.

\section{Methods}

\section{Participants}

A total of 98 youth aged 7-17 years participated in the study. Seventy-eight participants were in the clinical sample and 20 participated as part of a nonclinical sample. A lower age limit of seven years was selected because, in order to participate, subjects needed to be able to describe their thoughts and anticipate their actions well enough to respond accurately to the measures being administered. ${ }^{20}$ The upper age limit of 17 years was selected to provide a broad enough age range to examine age-related differences. This upper age limit is also consistent with previous research examining the types of variables utilized in this study. ${ }^{13}$

\section{Clinical sample}

The clinical sample ( $\mathrm{n}=78,29$ boys, 49 girls, mean age 11.86, standard deviation $[\mathrm{SD}] \pm 3.11$ years) was comprised of youth recruited via consecutive clinical referrals to a University-based research clinic and met Diagnostic and Statistical Manual, Fourth Edition, Text Revision (DSM-IV-TR) ${ }^{21}$ criteria for a principal anxiety disorder diagnosis of GAD $(n=20)$, OCD $(\mathrm{n}=18), \operatorname{SOC}(\mathrm{n}=20)$, or SAD $(\mathrm{n}=20)$. Diagnoses were made using the Anxiety Disorders Interview Schedule, Child and Parent Versions (ADIS-IV-C/P). ${ }^{22}$ Young people were included even if they met criteria for additional, comorbid diagnoses (eg, other anxiety, depressive, or behavioral disorders) that were less severe and/or interfering. Research has found that comorbidity amongst youth with anxiety disorders is extremely common, ${ }^{23,24}$ and some studies suggest that it is possible to examine differences between youth grouped together by a primary anxiety disorder diagnosis despite symptom overlap with other disorders. ${ }^{14}$ In the present study, $60 \%$ of the subjects in the clinical sample had at least one additional anxiety disorder, $12 \%$ met criteria for an additional depressive disorder, and 13\% met criteria for an additional behavioral, attentional, or impulse control disorder, or learning disability. Youth with comorbid pervasive developmental disorder, mental retardation, bipolar disorder, and psychosis were excluded from the present study, as these 
were general exclusion criteria for the larger research clinic in which this investigation was conducted.

\section{Nonclinical sample}

Twenty additional youth from the community (seven boys, 13 girls), aged 7-17 (mean $12.41 \pm 3.02$ ) years served as participants in a nonclinical control group. These youth were primarily recruited from advertisements posted on an Internet bulletin board and from fliers posted in the community. Inclusion criteria for nonclinical participants included: no diagnosis of a mental disorder according to an abbreviated version of the parent-report form of the ADIS-IV-C/P or one or more subclinical diagnoses of a mental disorder on the ADIS-IV-C/P, but with a Clinician Severity Rating (CSR) $<4$ for any disorder assigned. Eight $(40 \%)$ of the nonclinical participants had no mental disorder, while 12 (60\%) had subclinical symptoms of one or more mental disorder(s) and, of these, seven $(60 \%)$ had CSRs of 1 or 2 .

Demographic characteristics of the diagnostic groups, including participants' age, gender, ethnicity, household income, parents' marital status and parents' education were examined (listed in Table 1). There were no statistically significant differences between the diagnostic groups for age, gender, reported household income, or parents' education level. With regard to family characteristics, the parents of subjects in both groups were primarily Caucasian, affluent, and college-educated.

\section{Procedure}

Potential participants who met the criteria for participation in the study were approached after receiving feedback about the results of their diagnostic assessment at the clinic. After giving informed consent and assent to be in the study, those who agreed to participate completed a set of self-report measures. For youth in the nonclinical sample who responded to Internet and community advertisements, the study session was scheduled either at the clinic or at the participants' home, with nine parents $(45 \%)$ choosing to do the study at home. After the receipt of informed consent/assent, the participant's mother was administered an abbreviated version of the parent ADIS-IV-C/P form. None of the youth recruited to the nonpatient group met criteria for an anxiety or mood disorder at a clinical level. After inclusion criteria were established, the child or adolescent was asked to complete the same measures as the clinical sample (the order of the measures was counterbalanced).

\section{Measures}

\section{Anxiety Disorders Interview Schedule, Child and Parent Versions (ADIS-IV-C/P)}

This is a semistructured clinical interview for the diagnosis of childhood anxiety and related disorders. Youth and their parents are interviewed separately by a single interviewer, and diagnoses are based on composite information from both interviews. A CSR is assigned to each anxiety disorder diagnosis; CSRs range from zero (absent) to eight (very severe), with a CSR of four or higher representing a clinical diagnosis. Research demonstrates that the ADIS-IV-C/P has good inter-rater and test-retest reliability, ${ }^{25,26}$ with reports of kappa coefficients for specific anxiety disorder diagnoses assigned using the ADIS-IV-C/P. ${ }^{27,28}$ Inter-rater reliability

Table I Demographic characteristics of diagnostic groups of participants

\begin{tabular}{|c|c|c|c|c|c|}
\hline & GAD & OCD & soc & SAD & NONP \\
\hline & $(n=20)$ & $(n=18)$ & $(n=20)$ & $(n=20)$ & $(n=20)$ \\
\hline Mean age (years) & 11.37 & 13.45 & 14.25 & 8.51 & 12.46 \\
\hline SD & $\mathrm{I} .77$ & 2.86 & 2.52 & 1.37 & 12.42 \\
\hline Number of females & 14 & 7 & 10 & 13 & 13 \\
\hline Number of males & 6 & 7 & 10 & 7 & 7 \\
\hline Caucasian (\%) & 100 & 94 & 100 & 100 & 80 \\
\hline African American (\%) & 0 & 6 & 0 & 0 & 0 \\
\hline Hispanic/Latino (\%) & 0 & 0 & 0 & 0 & 10 \\
\hline Asian American (\%) & 0 & 0 & 0 & 0 & 10 \\
\hline Median household income $(\$)$ & 87,500 & 100,000 & 77,500 & 115,000 & 110,000 \\
\hline Parents married (\%) & 85 & 84 & 80 & 100 & 85 \\
\hline Parents divorced (\%) & 5 & 10 & 15 & 0 & 5 \\
\hline Parents separated (\%) & 10 & 0 & 0 & 0 & 5 \\
\hline Never married (\%) & 0 & 6 & 0 & 0 & 5 \\
\hline Same-sex partnership (\%) & 0 & 0 & 5 & 0 & 0 \\
\hline Parent modal education level & BA & BA & BA & BA & BA \\
\hline
\end{tabular}

Abbreviations: BA, Bachelor's degree; GAD, generalized anxiety disorder; OCD, obsessive-compulsive disorder; SOC, social phobia; SAD, separation anxiety disorder; NONP, nonpatients. 
analyses for 60 subjects at this treatment site indicated good inter-rater agreement $(\kappa=0.866)$ regarding diagnostic impression (ie, what was assigned as primary diagnosis) and clinical severity (Pearson product-moment correlation $r=0.615)$. In addition, support for convergent validity of the ADIS-IV-C/P has been found ${ }^{29}$ in a study reporting high correlations between symptom ratings for the social phobia, separation anxiety, and panic disorder sections of the ADISIV/C-P and corresponding scales of the Multidimensional Anxiety Scale for Youth. ${ }^{30}$ An abbreviated version containing select subsections of the parent form of the ADIS-IV-C/P was administered to parents of community participants. This version was selected to allow for a slightly briefer screening of anxiety symptomatology (along with psychosis, bipolar disorder. and developmental disorders).

\section{Metacognitions Questionnaire for Youth-Child Version (MCQ-C)}

To measure young people's levels of cognitive monitoring, positive meta-worry, negative meta-worry, and SPR beliefs, subscales of the MCQ-C were administered. The MCQ-C is an adaptation of the MCQ-A. ${ }^{13}$ The MCQ-C differs from the MCQ-A in that it is intended to be applicable for a broader age range (children as well as adolescents). Briefly, the MCQ-C is a 24-item scale designed for young people aged 7-17 years. It is a multicomponent measure assessing a range of metacognitive beliefs and monitoring tendencies in youth, including intrusive thinking, worry, and the tendency to monitor thought processes. The MCQ-C is comprised of four subscales that were originally in the MCQ-A, with titles of subscales modified slightly. Results of a confirmatory factor analysis indicate that these subscales (positive meta-worry, negative meta-worry, SPR beliefs, and cognitive monitoring) reflect four valid factors and that the factor structure of the MCQ-C is comparable with the MCQ-A. ${ }^{12}$ The MCQ-C consists of a series of statements, eg, "I try hard to keep track of the thoughts in my head," (cognitive monitoring). Participants are asked to indicate how much they agree with each statement on a four-point Likert-type scale, labeled "do not agree" at one extreme, and "agree very much" at the other. There are six items on each subscale, and the sum of the items on the cognitive monitoring, negative meta-worry, positive meta-worry, and SPR subscales were used in the analyses as measures of these constructs. The total score on the MCQ-C was also used in the analyses as a general measure of metacognitive awareness and processes. The MCQ-C has a Flesch-Kincaid reading grade level of 2.0 .
The MCQ-C has good internal consistency reliability. Coefficient alphas for the MCQ-C observed in this investigation were 0.87 for the total scale, 0.86 for positive meta-worry, 0.75 for negative meta-worry, 0.64 for SPR beliefs, and 0.75 for cognitive monitoring, respectively, for the entire sample. For the clinical sample, coefficient alphas were 0.89 for the total scale, 0.89 for positive meta-worry, 0.74 for negative metaworry, 0.69 for SPR beliefs, and 0.75 for cognitive monitoring. Coefficient alphas for the nonclinical sample were 0.71 for the total scale, 0.60 for positive meta-worry, 0.76 for negative meta-worry, 0.58 for SPR beliefs and 0.74 for cognitive monitoring. In addition, Bacow et $\mathrm{al}^{12}$ provided support for the concurrent validity of the measure. MCQ-C beliefs were positively associated with self-reported measures of excessive worry (PSWQ-C) ${ }^{31}$ and depression (CDI). ${ }^{32}$

\section{Measure of worry content}

A measure was needed to assess for worry content or the amount of attention paid to worries of various content areas, ie, Type 1 worry $^{10}$ to test for the independent contributions of metacognition and worry content in examining diagnostic group differences. In previous research with adults, the Anxious Thoughts Inventory (AnTI) ${ }^{33}$ has been utilized to capture the construct of worry content. This is a 22-item multidimensional measure of trait worry, consisting of three factorially reliable subscales of social worry, health worry, and meta-worry (worry about worry). Because no such measure exists for youth, for the purpose of the present study, we elected to utilize an available measure encapsulating different worry content areas for young people. We selected the content areas represented in the GAD section of the ADIS-IV-C (ADIS-C) and parent report of the shorter version of the ADIS-IV-C/P (Mini ADIS-P) for the nonclinical sample to capture worry content. The GAD section assesses for the severity of eight content domains of worry, ie, school, performance, social and interpersonal matters, perfectionism, health (self), health (others), family matters, and current events. Youth or their parents were asked to rate how much the child or adolescent worried about each topic on a scale from 0 to 8 . The total score of all worry domain ratings in this section (maximum score 64) comprised the estimate of worry content. For example, if a child or adolescent or adolescent reported worrying about all eight topics and gave a rating of 8 for each, she received a score of 64 . Although this study is the first to capture worry content with youth using this approach, this measure was intended to differ functionally from measures of proneness to generalized worry, eg, Penn State Worry Questionnaire (PSWQ-C) $)^{31}$ in that it specifically includes worry content areas. There was a low $(r=0.42)$, but significant, correlation 
between the worry content measure and the PSWQ-C for the clinical group only, suggesting some overlap between the two. However, Wells and Carter ${ }^{1}$ also found a significant correlation between the AnTI and the adult $\operatorname{PSWQ}^{34}$ ( $r=0.69$ for social worry and $r=0.60$ for health worry), suggesting that it is difficult to create a "pure" measure of worry content, given the fact that worry about several different topic areas may also indicate some degree of worry excessiveness.

\section{Results \\ Statistical plan}

These analyses represent additional tests that were performed on the same data set from our original study. The original study explored between-group differences in metacognitive processes by examining differences between the clinical group as a whole $(n=78)$ and the nonclinical sample $(n=20)$. In contrast, the present analyses represent an investigation of within-group differences in the entire sample $(n=98)$. Specifically, the multivariate analysis of variance (MANOVA) technique was used to compare nonclinical participants with participants from the clinical sample with principal diagnoses of GAD $(n=20)$, OCD $(n=18)$, SOC $(n=20)$, and SAD $(n=20)$. Thus, participants were grouped by diagnostic status for the purpose of the present investigation, and results of these analyses are reported below.

\section{Descriptive statistics}

Chi-square analyses revealed that there were no gender or ethnicity differences amongst youth with GAD, OCD, SOC, or SAD. With regard to age differences, results of a one-way ANOVA comparing ages of participants with each diagnosis (with age treated as a continuous variable) showed that the overall Welch $F$ was significant $(F, 3,36.47)=28.8$, $\mathrm{MS}_{\mathrm{E}}=4.44, p<0.001$. Post hoc comparisons using the Games-Howell procedure revealed that clinical participants with $\mathrm{SAD}(\mathrm{M}=8.59, \mathrm{SD}=1.36)$ were significantly younger than those with $\mathrm{GAD}(\mathrm{M}=11.37, \mathrm{SD}=1.77), \mathrm{OCD}$ $(\mathrm{M}=13.70, \mathrm{SD}=2.66)$, and $\mathrm{SOC}(\mathrm{M}=14.28, \mathrm{SD}=2.46)$. These comparisons also revealed that clinical participants with SOC $(\mathrm{M}=14.28, \mathrm{SD}=2.46)$ and with $\mathrm{OCD}(\mathrm{M}=13.70$, $\mathrm{SD}=2.66)$ were significantly older than young people with GAD $(\mathrm{M}=11.37, \mathrm{SD}=1.77)$ and $\mathrm{SAD}(\mathrm{M}=8.59$, $\mathrm{SD}=1.36)$. Descriptive results are presented in Table 1 .

\section{Selection of covariates}

Given the age-based findings above, we explored whether age met the statistical criteria to be selected as a covariate in this investigation. Although there were some between-group differences with regard to age and diagnostic status, age was not significantly correlated with any of the main outcome measures (the metacognitive variables of interest) and thus did not meet the full criteria for selection as a covariate. Of note, the differences in mean ages between some diagnostic groups in this study are not unexpected, given age-based prevalence rates finding that $\mathrm{SAD}$ is more common in younger youth and that OCD and SOC tend to have their onset in early adolescence, and this pattern is often found in the childhood anxiety disorders. ${ }^{35,36}$ Worry content was also evaluated for possible selection as a covariate given its theoretical overlap with the metacognitive variables of interest. Comparisons of worry content amongst diagnostic groups showed that significant differences emerged among the groups; Welch $F(4,44.88)=4.39$, $\mathrm{p}<0.01$. Participants with GAD and OCD obtained higher scores than nonpatients, and participants with GAD and SOC obtained higher scores than those with SAD. Further, worry content was significantly correlated with four of the five metacognitive variables (all but positive meta-worry).

\section{Analysis of diagnostic group differences in metacognitive processes}

A multivariate analysis of covariance (MANCOVA) was conducted to examine group differences amongst youth with GAD, OCD, SOC, and SAD and nonclinical participants on the metacognitive variables with worry content as the covariate, followed by Sidak post hoc comparisons to locate pair-wise differences. Untransformed covariate-adjusted mean scores and SDs for each group are displayed in Table 2, and the statistics represented in the analysis are outlined in Table 3. On the MCQ-C, the diagnostic groups (GAD, OCD, SOC, SAD, nonpatients) did not differ in their endorsement of SPR beliefs, or positive or negative meta-worry. However, the overall F-test was significant for cognitive monitoring: $\mathrm{F}(4,92)=3.64, p<0.01$. Post hoc tests using the Sidak procedure revealed that nonpatients reported being significantly more aware of their thoughts than their counterparts with SAD. In addition, there was a trend approaching significance $(p<0.06)$ suggesting that nonpatients were also marginally significantly more aware of their thinking processes than youth with GAD. The covariate-adjusted mean scores on cognitive monitoring for the SAD and GAD groups were 12.95 and 13.63, respectively, while the covariate-adjusted mean score for the comparison group was 17.37 .

\section{Measurement of effect sizes}

Given the relatively smaller sample sizes of the diagnostic groups in this study, we examined the effect sizes of the 
Table 2 Means, standard deviations and effect sizes for dependent variables by diagnostic group

\begin{tabular}{|c|c|c|c|c|c|c|c|}
\hline Measure & $\begin{array}{l}\text { GAD } \\
(n=20)\end{array}$ & $\begin{array}{l}\text { OCD } \\
(n=18)\end{array}$ & $\begin{array}{l}\text { SOC } \\
(n=20)\end{array}$ & $\begin{array}{l}\text { SAD } \\
(n=20)\end{array}$ & $\begin{array}{l}\text { NONP } \\
(n=20)\end{array}$ & $\begin{array}{l}\text { Partial eta } \\
\text { squared }\end{array}$ & Sig \\
\hline Worry content & $\begin{array}{l}21.15 \\
(13.39)\end{array}$ & $\begin{array}{l}14.50 \\
(15.76)\end{array}$ & $\begin{array}{l}19.15 \\
(16.4 I)\end{array}$ & $\begin{array}{l}9.75 \\
(8.96)\end{array}$ & $\begin{array}{l}5.40 \\
(4.98)\end{array}$ & 0.19 & n.s. \\
\hline MCQ-C total score & $\begin{array}{l}51.26 \\
(14.98)\end{array}$ & $\begin{array}{l}50.78 \\
(14.43)\end{array}$ & $\begin{array}{l}49.12 \\
(8.81)\end{array}$ & $\begin{array}{l}43.30 \\
(11.27)\end{array}$ & $\begin{array}{l}50.15 \\
(8.56)\end{array}$ & 0.08 & n.s. \\
\hline Positive meta-worry & $\begin{array}{l}9.26 \\
(4.23)\end{array}$ & $\begin{array}{l}8.56 \\
(4.00)\end{array}$ & $\begin{array}{l}9.00 \\
(3.56)\end{array}$ & $\begin{array}{l}8.40 \\
(3.80)\end{array}$ & $\begin{array}{l}10.15 \\
(2.91)\end{array}$ & 0.06 & n.s. \\
\hline Negative meta-worry & $\begin{array}{l}13.89 \\
(4.48)\end{array}$ & $\begin{array}{l}13.94 \\
(4.91)\end{array}$ & $\begin{array}{l}13.84 \\
(3.72)\end{array}$ & $\begin{array}{l}13.15 \\
(4.37)\end{array}$ & $\begin{array}{l}12.50 \\
(4.11)\end{array}$ & 0.02 & n.s. \\
\hline SPR beliefs & $\begin{array}{l}12.26 \\
(5.17)\end{array}$ & $\begin{array}{l}12.67 \\
(4.04)\end{array}$ & $\begin{array}{l}11.47 \\
(2.76)\end{array}$ & $\begin{array}{l}10.05 \\
(3.12)\end{array}$ & $\begin{array}{l}11.05 \\
(2.46)\end{array}$ & 0.05 & n.s. \\
\hline Cognitive monitoring & $\begin{array}{l}15.83 \\
(4.57)\end{array}$ & $\begin{array}{l}15.44 \\
(4.12)\end{array}$ & $\begin{array}{l}15.37 \\
(3.91)\end{array}$ & $\begin{array}{l}12.50 \\
(4.36)\end{array}$ & $\begin{array}{l}16.45 \\
(4.02)\end{array}$ & 0.14 & $\begin{array}{l}\text { NONP > SAD, } \\
\text { GAD }\end{array}$ \\
\hline
\end{tabular}

Abbreviations: GAD, generalized anxiety disorder; OCD, obsessive-compulsive disorder; SOC, social phobia; SAD, separation anxiety disorder; NONP, nonpatients; PSWQ-C, Penn State Worry Questionnaire for Children; CDI, Children's Depression Inventory; MCQ-C, Metacognitions Questionnaire for Children; SPR, superstition, punishment and responsibility; Sig, significance; n.s., not significant.

dependent variables (Table 2). The effect sizes for worry content and cognitive monitoring were considerably large, and the effect sizes for positive meta-worry, SPR beliefs, and the total score on the MCQ-C were medium. ${ }^{37}$ The effect size for negative meta-worry was small. These results indicate that, with greater statistical power, significant differences for the variables with medium and large effect sizes (all but negative meta-worry) would likely emerge.

\section{Discussion}

The goal of this study was to report additional findings from our initial investigation and examine diagnostic differences in metacognition between youth with four categories of principal anxiety disorder diagnoses and healthy controls (eg, nonpatients). Research with adults suggests that certain metacognitive constructs may be particularly relevant for specific anxiety disorder diagnoses, such as the link between negative meta-worry and $\mathrm{GAD},{ }^{7}$ and between cognitive monitoring and $\mathrm{OCD} .{ }^{8}$ This question has not been examined previously in the literature with youth, and results from the current study suggest that it may be presumptuous to assume that identical relationships exist for younger individuals.
Our study results were to a certain extent rather unexpected and surprising. Rather than confirming that the metacognitive model of GAD is fully applicable to youth, the results from this investigation suggest a very different set of patterns for our sample of youth. Our results indicated that with worry content held constant, nonpatient participants who did not meet criteria for any clinical anxiety disorder reported being more aware of their thoughts than participants with SAD. This study also found that young people who were nonpatients also tended to be more aware of their thoughts than youth with GAD, as reflected by a trend approaching statistical significance. This, while also unexpected, is consistent with previous findings using the MCQ-C, specifically that nonpatients as a whole reported being more aware of their thoughts than youth in a clinical sample with a range of anxiety disorder diagnoses. ${ }^{12}$

This finding could certainly be related to the fact that nonpatients were, on average, older than youth with both $\mathrm{SAD}$ and GAD (these two groups were the youngest) and that adolescents received higher scores on the MCQ-C than younger children. It is important to note that cognitive processes, such as rumination and worry, tend to be more

Table 3 Multivariate and univariate analyses of variance for diagnostic group differences in metacognitive processes

\begin{tabular}{|c|c|c|c|c|c|c|c|}
\hline & \multicolumn{2}{|c|}{ Multivariate } & \multicolumn{5}{|l|}{ Univariate } \\
\hline & df & $\mathbf{F}^{\mathbf{a}}$ & $\begin{array}{l}\text { MCQ-C } \\
\text { total score }\end{array}$ & $\begin{array}{l}\text { Positive }^{\mathrm{b}} \\
\text { meta-worry }\end{array}$ & $\begin{array}{l}\text { Negative }^{b} \\
\text { meta-worry }\end{array}$ & $\begin{array}{l}\text { SPR }^{\mathbf{b}} \\
\text { beliefs }\end{array}$ & $\begin{array}{l}\text { Cognitive }^{\mathrm{b}} \\
\text { monitoring }\end{array}$ \\
\hline $\begin{array}{l}\text { Diagnostic } \\
\text { group }\end{array}$ & 4 & $1.64 *$ & 2.00 & 1.45 & 0.51 & 1.09 & $3.64 * *$ \\
\hline $\begin{array}{l}\text { Worry } \\
\text { content }\end{array}$ & I & $5.43 * *$ & $19.62 * *$ & 3.41 & $23.47 * *$ & $5.08 *$ & $10.72 * *$ \\
\hline
\end{tabular}

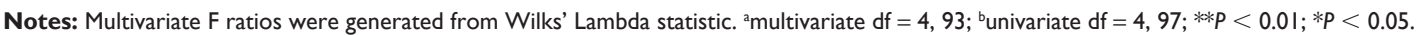

Abbreviations: MCQ-C, Metacognitions Questionnaire for Children; SPR, superstition, punishment and responsibility. 
advanced in older youth. ${ }^{20}$ However, there was no significant difference in ages between the nonpatients and the groups of children with anxiety disorders.

One possible explanation for this pattern is the suggestion by Cartwright-Hatton et $\mathrm{al}^{13}$ that anxious youth (and those with GAD and SAD in particular) use the coping strategy of cognitive avoidance (ie, avoidance of thoughts or threatening mental imagery that are upsetting) when worrisome thoughts enter their mind. This could certainly be true of GAD, a disorder in which avoidance is a common coping strategy, and which young individuals report to be interfering and distressing. ${ }^{11,31}$ Consequently, worry exposure is occasionally used as a cognitive-behavioral intervention to help individuals learn how to face their worries over and over until the emotional salience of the worry decreases. ${ }^{38}$ Although we did not find that young people with GAD reported greater amounts of negative meta-worry than nonpatients in this study, several previous studies in youth have found that youth with GAD find worry to be an aversive process and that negative beliefs about worry are certainly present and common at younger ages. ${ }^{13,15,16}$ It is possible that youth with GAD in this study who demonstrated relatively lower awareness of thoughts were less likely to endorse very strong beliefs about the danger and meaning of their cognitive processes. Furthermore, $\mathrm{SAD}$, in comparison to GAD, may be considered less of a "worry-based" disorder in which the focus is generally on a more present-focused fear of separation from parents and being alone rather than intrusive, worrisome thoughts geared towards future events. ${ }^{36}$ In this light, the lower levels of cognitive monitoring reported by these participants could be a function of the disorder rather than the use of an avoidant coping strategy per se.

It is also important to note that these two groups (GAD and SAD) also had the lowest PSWQ-C and CDI scores of the clinical participants. It could be that cognitive monitoring serves a different function for youth with different anxiety disorders, with awareness of thoughts possibly less specific to GAD and SAD, particularly when fewer internalizing symptoms are reported by these groups.

This unexpected finding could also reflect genuine increases in cognitive monitoring amongst nonpatients. Cognitive monitoring encompasses the ability to "read" one's own mental states and assess accurately how that state will affect present and future performance on mental activity tasks. ${ }^{39}$ It is possible that healthy controls have a more "normative" awareness of their thought processes, characterized by a healthy attentiveness to thoughts that occur in the stream of consciousness. This may in part explain why youth without anxiety disorder diagnoses endorsed relatively greater amounts of cognitive monitoring than two of the four anxiety disorder groups. Michael Vasey, a researcher on cognitive development and worry in young individuals, has suggested that anxious youth may lack the metacognitive awareness that they often worry about things that do not bother others and that they may be poor at recognizing and monitoring their level of affective arousal. He further suggests that young people with anxiety disorders fail to recognize when they are engaged in anxious self-talk, which could prevent them from engaging in selfregulatory mechanisms at the optimal time. ${ }^{40}$ Additional research is needed to explore at what level awareness of thoughts is normative, with the possibility that too much (eg, excessive rumination about thoughts) or too little (ie, avoidance and difficulty with metacognitive awareness of anxious cognitions) awareness may relate to the experience of anxiety at a young age.

While further research is needed to replicate these findings and to explore the additional hypotheses emerging from these data, particularly given the surprising nature of the results, the present study's findings about the metacognitive processes of anxious children and adolescents has both theoretic and clinical implications. The level of cognitive specificity characterizing different types of emotional disorders in childhood, and anxiety disorders, in particular, is not fully understood. However, research with adults ${ }^{4}$ supports the view that differences in cognition are evident even amongst the different subtypes of anxiety disorders. In the case of adult metacognition, the available literature suggests that functionally distinct metacognitive processes make independent contributions to specific anxiety disorders. Results from this study suggest that this may be at least partially the case for anxious youth. At least in this sample, it appears that cognitive monitoring may have particular salience for youth with GAD and SAD in that they have lower levels than healthy controls. If this is the case, then clinicians treating these particular disorders may benefit from considering whether helping youth become more aware of their cognitions (eg, using mindfulness techniques or metacognitive therapy) would be beneficial for treatment, as in the case of the cognitive-behavioral technique of cognitive restructuring (in which youth are asked to identify and challenge dysfunctional thoughts). In the case of OCD, a metacognitive therapy has been piloted with young people aged $8-17$ years. ${ }^{41}$ In this particular treatment, the therapist helps the client change faulty metacognitive strategies (ie, diminishing selective attention to thoughts via behavioral experiments). It would be 
interesting to see whether similar strategies could be used to help youth identify their automatic thoughts and worries to facilitate cognitive therapy.

Limitations of this study should be taken into consideration when interpreting the findings. Firstly, it was difficult to understand the relative influence of age as separate from diagnosis in our study, given the overlap of the two, both in our sample and in general. Prevalence rates for anxiety disorders indicate that $\mathrm{SAD}$ is much more common in younger youth and that it is rare for SAD to be diagnosed in adolescence. Future research using a larger sample with a wider range of ages would enable investigators to separate better the independent contributions that age and diagnosis make to metacognitive processes. Furthermore, given the diagnostic composition of our sample, it was not possible in this study to exclude participants with mixed diagnoses of depression, anxiety, and/or behavioral disorders, or to exclude participants with additional anxiety disorder diagnoses. Symptom overlap between the groups may have possibly obscured diagnostic group differences and quite possibly influenced the results. Further, the fact that $40 \%$ of the nonclinical sample had some subclinical symptoms of anxiety may explain some of the similarities between the clinical and nonclinical groups. However, it was not possible to control statistically for comorbidity in this context. ${ }^{42}$ As pointed out by Dalgeish et $\mathrm{al}^{14}$ regarding their investigation using multiple diagnostic groups, the comorbid diagnostic groups represent a conservative test of group differences despite the possibility that symptom overlap would have diluted any effects. And perhaps most importantly, comorbid groups show the reality of how these disorders present, which is rarely in a single diagnosis. ${ }^{23,24}$

Future research should attempt to replicate the findings of the present study in youth with additional anxiety disorders (ie, post-traumatic stress disorder, panic disorder) and continue to consider sthe role of related cognitive constructs (ie, thought control strategies, intolerance of uncertainty, thought-action fusion) in young people with internalizing disorders. Given the developmental differences between youth and adults, examining metacognitive processes in the context of anxiety represents a particularly fruitful avenue for improving psychologic treatments of anxious youth. This approach could also enable us to achieve a better conceptualization of the emotional problems of youth, particularly those characterized by both intrusive thought and cognitive avoidance. Anxiety disorders that include hyperfocus on thoughts or avoidance of aversive cognitive stimuli (ie, GAD and OCD) are often more difficult to treat, regardless of age. There is much to be gained by obtaining further insight into the cognitive processes of youth with anxiety disorders, and particularly the way in which both reduced awareness of and hyperfocus on thoughts may relate to youths experience of anxiety and worry.

\section{Disclosure}

The authors report no conflict of interest in this work.

\section{References}

1. Wells A, Cater K. Preliminary tests of a cognitive model of generalized anxiety disorder. Behav Res Ther. 1999;37:585-594.

2. Wells A. Emotional disorders and metacognition: Innovative cognitive therapy. Chichester: Wiley; 2000.

3. Cartwright-Hatton S, Wells A. Beliefs about worry and intrusions: The Meta-Cognitions Questionnaire and its correlates. J Anxiety Disord. 1997;11:279-296.

4. Wells A. Metacognition and worry: A cognitive model of generalized anxiety disorder. Behav Cogn Psychother. 1995;23:301-320.

5. Wells A, Papageorgiou C. Relationships between worry, obsessivecompulsive symptoms and meta-cognitive beliefs. Behav Res Ther. 1998;36:899-913.

6. Wells A, Sembi S. Metacognitive therapy for PTSD: A preliminary investigation of a new brief treatment. J Behav Ther Exp Psychiatry. 2004;35:307-318.

7. Wells A. The metacognitive model of GAD: Assessment of metaworry and relationship with DSM-IV generalized anxiety disorder. Cogn Ther Res. 2005;29:107-121.

8. Janeck AS, Calamari JE, Riemann BC, Heffelfinger SK. Too much thinking about thinking? Metacognitive differences in obsessive-compulsive disorder. J Anxiety Disord. 2003;17:181-195.

9. Roussis P, Wells A. Psychological factors predicting stress symptoms: Metacognition, thought control, and varieties of worry. Anxiety Stress Coping. 2008;21:213-225.

10. Wells A, Carter K. Further tests of a cognitive model of generalized anxiety disorder: Metacognitions and worry in GAD, panic disorder, social phobia, depression and nonpatients. Anxiety Stress Coping. 2001; 32:85-102.

11. Comer J, Kendall P, Franklin ME, Hudson JL, Pimental SS. Obsessing/ worrying about the overlap between obsessive-compulsive disorder and generalized anxiety disorder in youth. Clin Psychol Rev. 2004;24: 663-683.

12. Bacow TL, Pincus DP, Ehrenreich JT, Brody LR. The Metacognitions Questionnaire for Children: Development and validation in a clinical sample of children and adolescents with anxiety disorders. $J$ Anxiety Disord. 2009;23:727-736.

13. Cartwright-Hatton S, Mather A, Illingworth JB, Harrington R, Wells A. Development and preliminary validation of the meta-cognitions questionnaire - adolescent version. J Anxiety Disord. 2004;18:411-422.

14. Dalgeish T, Taghavi R, Neshat-Doost H, et al. Patterns of processing bias for emotional information across clinical disorders: A comparison of attention, memory and prospective cognition in children and adolescents with depression, generalized anxiety and posttraumatic stress disorder. J Clin Child Adolesc Psychol. 2003;32: $10-21$.

15. Szabo M, Lovibond PE. The cognitive content of thought-listed worry episodes in clinic-referred anxious and nonreferred children. $J$ Clin Child Adolesc Psychol. 2004;33:613-620.

16. Xifu Z. The relationship between metaworry of middle school students and generalized anxiety disorder. Acta Psychol Sinica. 2002;34: 284-288.

17. Matthews L, Reynolds S, Derisley J. Examining cognitive models of obsessive-compulsive disorder in adolescents. Behav Cogn Psychother. 2006;35:149-163. 
18. Jacobi DM. Thought appraisals, beliefs and obsessional symptoms: Relations between parental beliefs and child symptoms [Dissertation]. Chicago, IL: Chicago Medical School; 2004.

19. Silverman WK, La Greca A, Wasserstein S. What do children worry about? Worries and their relation to anxiety. Child Dev. 1995;66: 671-686.

20. Vasey MW, Crnic KA, Carter WG. Worry in childhood: A developmental perspective. Cognit Ther Res. 1994;18:529-549.

21. American Psychiatric Association. Diagnostic and Statistical Manual of Mental Disorders, Fourth Edition, Text Revision (DSM-IV-TR). Washington, DC: American Psychiatric Association; 2000.

22. Silverman WK, Albano AM. The Anxiety Disorders Interview Schedule for DSM-IV: Child and Parent Versions. San Antonio, TX: Psychological Corporation; 1996.

23. Brady EU, Kendall PC. Comorbidity of anxiety and depression in children and adolescents. Psychol Bull. 1992;111:244-255.

24. Lewinsohn PM, Zinbarg R, Seeley JR, Lewinsohn M, Sack WH. Lifetime comorbidity among anxiety disorders and between anxiety disorders and other mental disorders in adolescents. J Anxiety Disord. 1997;11:377-394.

25. Silverman WK, Eisen AR. Age differences in the reliability of parent and child reports of child anxious symptomatology using a structured interview. J Am Acad Child Adolesc Psychiatry. 1992;31:117-124.

26. Silverman WK, Nelles WB. The Anxiety Disorders Interview Schedule for Children. J Am Acad Child Adolesc Psychiatry. 1998;27: $772-778$.

27. Rapee RM, Barrett PM, Dadds MR. Reliability of the DSM-III-TR anxiety disorders using a structured interview: Interrater and parent-child agreement. J Am Acad Child Adolesc Psychiatry. 1994;33:984-992.

28. Silverman WK, Saavendra LM, Pina AA. Test-retest reliability of anxiety symptoms and diagnoses with the Anxiety Disorders Interview Schedule for DSM-IV: Child and Parent Versions. J Am Acad Child Adolesc Psychiatry. 2001;40:937-944.

29. Wood JJ, Piacentini JC, Bergman RL, McCracken J, Barrios V. Concurrent validity of the anxiety disorders section of the Anxiety Disorders Interview Schedule for DSM-IV: Child and Parent Versions. J Clin Child Adolesc Psychol. 2002;31:335-342.

30. March JS, Parker James DA, Sullivan K. The multidimensional anxiety scale for children (MASC): Factor structure, reliability and validity. J Am Acad Child Adolesc Psychiatry. 1997;36:554-565.
31. Chorpita BF, Tracey SA, Brown TA, Collica TJ, Barlow DH. Assessment of worry in children and adolescents: An adaptation of the Penn State worry questionnaire. Behav Res Ther. 1997;35:569-581.

32. Kovacs M. Rating scales to assess depression in school-age children. Acta Paedopsychiatr. 1981;46:305-315.

33. Wells A. A multi-dimensional measure of worry: Development and preliminary validation of the Anxious Thoughts Inventory. Anxiety Stress Coping. 1994;6:289-299.

34. Meyer TJ, Miller ML, Metzeger RL, Borkovec TD. Development and validation of the Penn State Worry Questionnaire. Behav Res Ther. 1990;28:487-595

35. Francis G, Last CG, Strauss CC. Expression of separation anxiety disorder - the roles of age and gender. Child Psychiatr Hum Dev. 1987;18:82-89.

36. Albano AM, Chorpita BF, Barlow DH. Childhood anxiety disorders. In: Mash EJ, Barkley RA, editors. Child Psychopathology. 2nd ed. New York, NY: Guilford Press; 2003.

37. Cohen J. Statistical Power Analysis for the Behavioral Sciences. 2nd ed. Hillsdale, NJ: Lawrence Erlbaum Associates; 1988.

38. Hoyer J, Beesdo K, Gloster AT, Runge J, Hofler M, Becker ES. Worry exposure versus applied relaxation in the treatment of generalized anxiety disorder. Psychother Psychosom. 2009;78: 106-115.

39. Wellman HM. Metamemory revisited. In: Chi MTH, editor. Contributions to Human Development: Trends in Memory Development Vol 2. Basel: Karger; 1983.

40. Vasey MW. Development and cognition in childhood anxiety: The example of worry. In: Ollendick TH, Prinz RJ, editors. Advances in Clinical Child Psychology, Volume 15. New York, NY: Plenum Press; 1993.

41. Simons M, Schneider S, Herpetz-Dahlmann B. Metacognitive therapy versus exposure and response prevention for pediatric obsessive-compulsive disorder. Psychother Psychosom. 2006;75: 257-264.

42. Miller GM, Chapman JP. Misunderstanding analysis of covariance. J Abnorm Psychol. 2001;110:40-48.

Fisher PL, Wells A. Experimental modification of beliefs in obsessivecompulsive disorder: A test of the metacognitive model. Behav Res Ther. 2005;43:821-829.
Psychology Research and Behavior Management

\section{Publish your work in this journal}

Psychology Research and Behavior Management is an international, peerreviewed, open access journal focusing on the science of psychology and its application in behavior management to develop improved outcomes in the clinical, educational, sports and business arenas. Specific topics covered include: Neuroscience, memory \& decision making; Behavior

\section{Dovepress}

modification \& management; Clinical applications; Business \& sports performance management; Social and developmental studies; Animal studies. The manuscript management system is completely online and includes a quick and fair peer-review system. Visit http://www.dovepress. com/testimonials.php to read real quotes from published authors. 